\title{
E-Health Alert System Using Breathing Gestures (Breath Print) Classifying
}

\author{
Suhiar Mohammed Zeki, University of Technology / Computer \\ Science Department. Email: 110121@uotechnology.edu.iq \\ Mobile: +9647823549971 \\ ORCID: http://orcid.org/0000-0001-8337-4013 \\ Publons: 1630103
}

URL: //suhairmohammed1.academia.edu

Received: 5/3/2020 Accepted: 27/4/2020 Published: $1^{\text {st }}$ May, 2020

\begin{abstract}
Background: Electronic Health is an emerging field in the intersection of medical informatics, public health and business, referring to health services and information delivered or enhanced through the Internet and related technologies.
\end{abstract}

Purpose: One of the major needs of the E-Health is how to provide alert feature in order to perform specific action based on this alert and using this alert to provide the required health care to the patient.

Method: In this paper proposed an alert system for the E-Health purposes is presented which is based the using of patient breathing signal. This breathing signal passed through three main processing phases which are the reading and plotting phase, feature extraction phase using Gammatore Frequency Cepstral Coefficients (GFCC), and Classifying phase using Gaussian Mixture Model (GMM). Then the classification result will be used to give alert to the E-Health system.

Results: E-Health field requires a lot of needs in order to work in correct way. One of these needs is the alert feature. In the proposed system the person breathing signal is used in order to provide this feature. The person breathing signal is processed in three phases which are: Reading and plotting phases using (MSR) and power, feature extraction phase using (GFCC), and classification phase uses (GMM). Then the classification result will be used to give alert to the doctors it is classified as sniff. 
Conclusion: The proposed E- health alert system using breathing gestures classifying found effective and may be used in hospital settings, especially intensive care and respiratory units and emergency ambulance settings.

Keywords: E-health, healthcare, biometric, Breath print, alert system

\section{Introduction}

E-health is defined as the application of Internet and other related technologies in the healthcare industry to improve the access, efficiency, effectiveness, and quality of clinical and business processes utilized by healthcare organizations, practitioners, patients, and consumers in an effort to improve the health status of patients [1]. E-health includes many dimensions [2]:

- Delivery of key information to healthcare partners.

- Provision of health information delivery services.

- Facilitation of interaction between providers and patients

- Facilitation of the integration of healthcare industry-related business processes.

- Both local and remote access to healthcare information.

- Support for employers and employees, payers and providers.

In recent times, it has become necessary to have a system that constantly monitors a patient's heart rate autonomously and then send an alert when readings are out of range to ensure healthcare is always provided on time [3].

Breath Print, a new behavioral biometric signature based on audio features derived from an individual's commonplace breathing gestures. Specifically, Breath Print uses the audio signatures associated with the three individual gestures: sniff, normal, and deep breathing, which are sufficiently different across individuals [4]. Figure (1) shows the spectrograms for the three breathing gestures. 

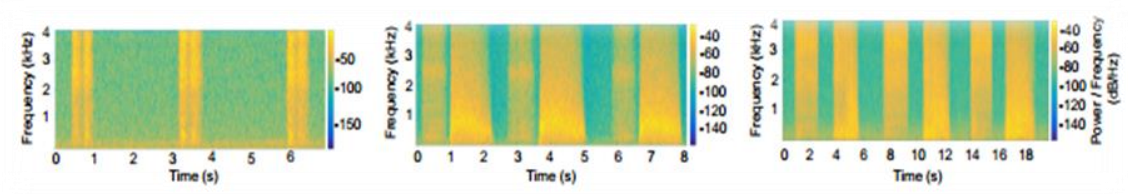

\section{a- Sniff b- normal breathing c- deep breathing}

\section{Figure (1): Spectrograms for the three breathing gestures.}

RMS, or Root Mean Square, is the measurement used for any time varying signal's effective value: It is not an "Average" voltage and its mathematical relationship to peak voltage varies depending on the type of waveform [5].

Power Frequency Disturbances describes events that are slower and longer lasting compared to electrical transients. Power Frequency Disturbances can last anywhere from one complete cycle to several seconds or even minutes [6]. Figure (2) shows the RMS and PowerFrequency Distribution.

Mel Frequency Cepstral Coefficients (MFCCs) are one of the most commonly used representations for audio speech recognition and classification [7]. A Gaussian Mixture Model (GMM) is a parametric probability density function represented as a weighted sum of Gaussian component densities. GMMs are commonly used as a parametric odal of the probability distribution of continuous measurements or features in a biometric system, such as vocal-tract related spectral features in a speaker recognition system. GMM parameters are estimated from training data using the iterative Expectation-Maximization (EM) algorithm or Maximum A Posteriori (MAP) estimation from a well-trained prior model [8].

\section{Related Work}

Kommey [3] presented a system to monitor heart rate and body temperature of a user and alert the user when these values are abnormal is proposed. Patient Medical Emergency Alert System (PMEAS) consists mainly of two components, a wearable hardware unit and an android application. The wearable unit contains sensors to monitor the heart rate and body temperature of the user, which are 
displayed on an LCD screen. These sensor values are transmitted to an android device via Bluetooth and are managed by the android application. An alert is sent to confidants via email or SMS when these values are abnormal.

Ufoaroh et al [9] proposed a heartbeat monitoring and alert system. The system uses a pulse sensor to measure the changes in the heartbeat of the user, and then calculates the heart rate in beats per minute (BPM). The system consists of a microcontroller unit, an LCD unit, a buzzer, a pulse sensor and a GSM module. The microcontroller calculates the BPM and displays it on the LCD screen. Each time the heart rate rises above or falls below a threshold, the buzzer sounds and an SMS message is sent via the GSM module with the current BPM value.

Abdulla et al [10] proposed a real time wireless health monitoring application. The system, a smart phone-based one, provides real time information about the medical status of a person. It is also able to send messages about the user's health status to a mentor for medical diagnosis and advice. The system consists of ECG electrodes, a temperature sensor, blood glucose sensor, blood pressure sensor and a Microsoft Surface Pro Tablet running LabVIEW software. The LabVIEW software displays the recorded values from the sensors for monitoring and analysis.

Subhani et al [11] proposed a GSM-based heart rate and temperature monitoring system. The system consists of a heart rate sensor, a GSM modem, a temperature sensor, an LCD display and a microcontroller. The heart rate sensor is made up of an LED and an LDR placed parallel to each other such that when the light from the LED hits the finger, the reflected rays are received by the LDR and the heart rate can be calculated based on the received signal at the LDR. The values recorded by the temperature and heart rate sensors are displayed on the LCD screen and are transmitted to some medical personnel before arriving at a hospital for timely diagnosis.

Other research group [12] proposed a patient monitoring system using GSM technology. The system measures the heart rate of the user from the index finger using an Infrared sensor. A temperature sensor also measures the body temperature and these values are displayed on the LCD screen. The system sounds an alarm when the recorded values exceed a threshold value set. These values are transmitted to a doctor via a GSM module. 




(a) Sniff - RMS

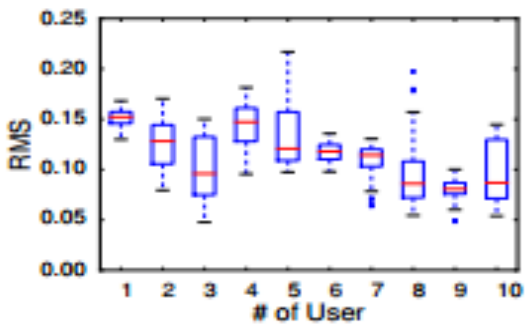

(c) Normal - RMS

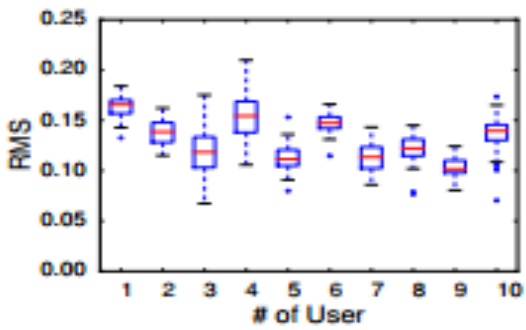

(e) Deep - RMS

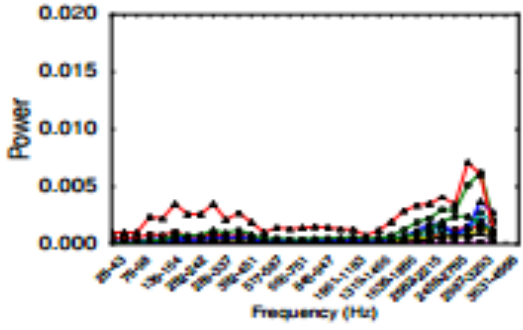

(b) Sniff - Power

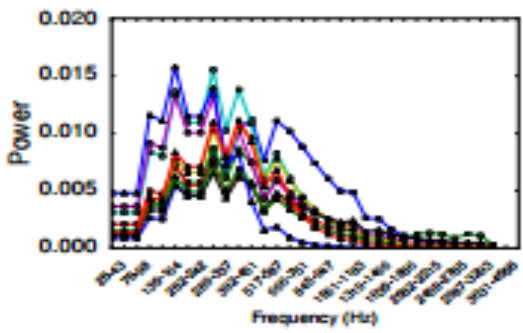

(d) Normal - Power

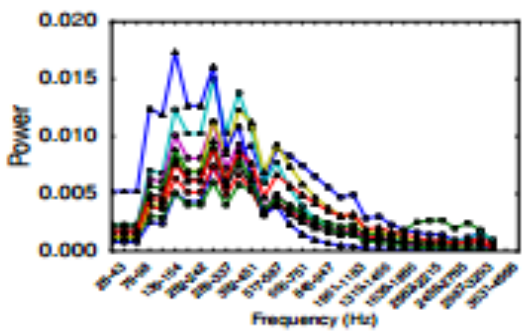

(f) Deep - Power

Figure (2): RMS and Power-Frequency Distribution

\section{The Proposed Alert System}

E-health plays important role in the modern care field. E-Health systems require a lot of needs, one of these important needs is how to provide alert feature in order to inform the doctors that there is emergency case about specific patient. In the proposed work specific alert system is presented based the using of patient breathing signal. In the proposed system the patient breathing signal is processed in order 
to be classified as sniff, normal, and deep breathing. This classification is used to give alert to the doctors about the patient emergency case. Figure (3) and algorithm (1) show the details of the proposed alert system steps.

\section{Reading the breathing signal}

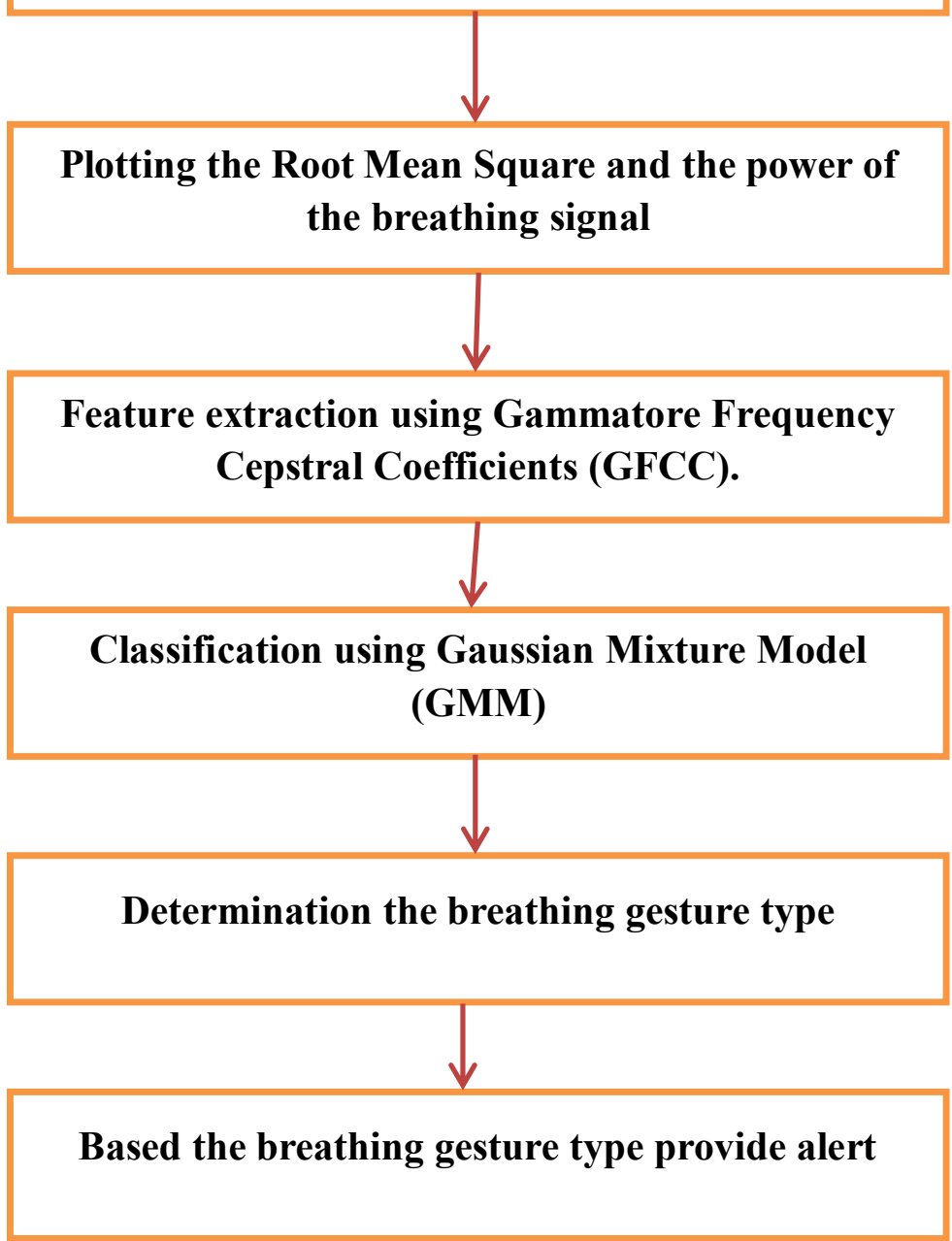

Figure (3): the details of the proposed alert system steps. 


\begin{tabular}{|l|}
\hline Algorithm (1): E-Health Alert System \\
\hline Input: Breathing signal. \\
\hline Output: Alert \\
\hline Begin \\
Step1: Reading the breathing signal. \\
Step2: Plotting the Root Mean Square (RMS) of the \\
amplitude of breathing signal. \\
Step3: Plotting the power. \\
Step4: Feature extraction using Gammatore Frequency \\
$\quad$ Cepstral Coefficient (GFCC). \\
Step5: Classification using Gaussian Mixture Model (GMM). \\
Step6: Determination of the breathing gesture type \\
6.1: If the breathing gesture is sniff give alert. \\
6.2: Else if the breathing gesture is normal don't give alert. \\
6.3: Else if the breathing gesture is deep don't give alert. \\
End
\end{tabular}

E-Health field requires a lot of needs in order to work in correct way. One of these needs is the alert feature. In the proposed system the person breathing signal is used in order to provide this feature. The person breathing signal is processed in three phases which are: Reading and plotting phases using (MSR) and power, feature extraction phase using (GFCC), and classification phase uses (GMM). Then the classification result will be used to give alert to the doctors it is classified as sniff.

\section{Conclusion}

The proposed E- health alert system using breathing gestures classifying found effective and may be used in hospital settings, especially intensive care and respiratory units and emergency ambulance settings. 


\section{References}

1. Marconi J. E-Health: Navigating the Internet for Health Information Healthcare. Advocacy White Paper. Healthcare Information and Management Systems Society, May, 2002.

2. Broderick M, Smaltz DH. E-Health Defined. Proceedings of Student Research Day, CSIS, Pace University, May 9th, 2003;16.116.11 .

3. Kommey B, Kotey SD, Opoku D. Patient Medical Emergency Alert System. Int J Appl Inform Systems (IJAIS), 2018;12(17):7-14.

4. Chauhan J, Hu Y, Seneviratne S, Misra A, Seneveratne A.

BreathPrint: Breathing Acoustics-based User Authentication.

MobiSys 17: Proceeding of the $15^{\text {th }}$ Annual International

Conference on a Mobile Systems, Applications and Services. June 2017:PP. 278-291.https://doi.org/10.1145/3081333.3081355

5. Tyco Electronic Corporation. Root Mean Square. Tyco Electronics Corporation, Crompton Instruments, Kennesaw, GA 30152. http://www.cromptonusa.com/RMS.pdf.

6. Kanani BD. Power Frequency Disturbances, Power Quality and Management. (2180911), Darshan. https://onlineaavedan.com/study_material/1546514142.pdf

7. Liu GK. Evaluating Gammatone Frequency Cepstral Coefficients with Neural Networks for Emotion Recognition from Speech. 2018. https://arxiv.org/pdf/1806.09010.pdf.

8. Chellappa R, Veeraraghavan A, Ramanathan N.. Gaussian Mixture Models. Gait Biometrics, Overview. In: Li S.Z., Jain A. (eds)

Encyclopedia of Biometrics. Springer, Boston, MA. 2009.

9. Ufoaroh SU, Oranugo CO, Uchechukwu ME. Heartbeat monitoring and alert system using GSM technology. Int J Engineer Res Gen Sci 2015;3(4):26-34.

10. Khaliq A, Abdullah AM, Saleh MU, Abbas MW, Maab H, Khan S. Real time wireless health monitoring system. IOSR Journal Electrical and Electronics Engineering (IOSR-JEEE)2014; 9(3: 3138.

11. Subhani SkM, Sateesh GNV, Chaitanya Ch, Prakash Babu G. Implementation of GSM Based Heart Rate and Temperature Monitoring System. Res J Engineer Sci 2013;2(4):43-45.

12. Sengeeth, K, Kumar RS, Kumar RS, Kumersan T, Venkatesh C. Patient Monitoring System Using GSM Technology. Int J Innovative Res Electric Electron Instrument Control Engineer 2016;4(3):1-3. 